\title{
Estenosis subglótica: Reporte de casos
}

\author{
Subglottic stenosis: Case report \\ Matías Gómez G1, Leandro Rodríguez H², Mónica Rojas 0², Carlos Tapia M¹.
}

\section{RESUMEN}

Introducción: La estenosis subglótica (ES) plantea un desafío clínico constante dado su alta morbimortalidad, diversidad de causas, localización, severidad y variedad de procedimientos terapéuticos.

objetivos: Determinar el perfil de pacientes con diagnóstico de ES, evaluar el tratamiento y sus resultados.

Material y método: Estudio descriptivo retrospectivo mediante revisión de fichas clínicas de pacientes con ES entre 2008 y 2011. Se describen las características demográficas, factores de riesgo, sintomatología, tipo y grado de estenosis, tratamiento, porcentaje de decanulación y complicaciones.

Resultados: 17 pacientes adultos fueron incluidos. Edad promedio: $51 \pm 14,37$ años. Sexo: 70,6\% femenino, 29,4\% masculino. El principal síntoma fue la disnea (76,5\%). La ES con compromiso sólo de partes blandas (88,2\%) y el grado III de severidad fueron predominantes. El tratamiento incluyó procedimientos endoscópicos (47\%) y quirúrgicos abiertos (41\%). La cirugía abierta, como único tratamiento o segundo paso presentó un porcentaje de decanulación menor al manejo endoscópico. La dilatación con broncoscopio presentó una tasa de éxito de 63\%. La tasa de decanulación general fue 58,8\%.

Conclusiones: La ES es una patología compleja. La baja tasa de sospecha, sintomatología inespecífica y el retraso en la consulta hace necesaria la creación de equipos de vía aérea multidisciplinarios para su pesquisa y manejo.

Palabras claves: Estenosis subglótica, porcentaje de decanulación, dilatación con broncoscopio, resección laringotraqueal.

\section{ABSTRACT}

Introduction: Subglottic stenosis (SE) pose a constant clinical challenge because it's high morbidity and mortality, diversity of causes, location, severity and variety of treatment procedures.

Aim: Determine the profile of patients with SE and evaluate the treatment and its results.

Material and method: Retrospective descriptive study by reviewing medical records of patients with SE between 2008 and 2011. Demographic characteristics, risk factors,

\footnotetext{
${ }^{1}$ Médicos Servicio Otorrinolaringología, Hospital del Salvador.

${ }^{2}$ Médico Residente Servicio Otorrinolaringología, Hospital del Salvador.

${ }^{3}$ Médico Cirujano, Universidad de Chile.
} 
symptoms, type and degree of stenosis, treatment, decannulation percentage and complications were described.

Results: 17 adult patients were included. Average age: $51 \pm 14.37$ years. Sex: $70.6 \%$ female and $29.4 \%$ male. The main symptom was dyspnea (76.5\%). The SE with only soft tissue involvement (88.2\%) and grade III severity were predominant. For treatment endoscopic procedures (47\%) and open surgery were used (41\%). Open surgical technique as unique treatment or as a second step, was less successful compared to endoscopic treatment. Bronchoscopic dilatation had a success rate of $63 \%$. The overall decannulation rate was $58.8 \%$.

Conclusions: The SE is a complex pathology. The low rate of suspicion, nonspecific symptoms and delay in medical consultation requires the creation of multidisciplinary teams for diagnosis and management.

Key words: Subglottic stenosis, percentage of decannulation, bronchoscopic dilatation, laryngotracheal resection.

\section{INTRODUCCIÓN}

La laringe y la tráquea son estructuras tubulares semirrígidas que tras una lesión, cicatrizan de manera concéntrica reduciendo su lumen ${ }^{1}$. La zona más estrecha y con mayor probabilidad de ser afectada es la región subglótica ${ }^{2}$, que en el adulto presenta un diámetro interior de $23 \mathrm{~mm}$. trasversal y $18 \mathrm{~mm}$. anteroposterior ${ }^{3}$.

La estenosis subglótica (ES) presenta una alta morbimortalidad para el paciente ${ }^{4}$. La variedad de causas, localización, grado de severidad, asociación a trastornos fonatorios y deglutorios junto a la diversidad de procedimientos y técnicas quirúrgicas para su tratamiento, plantean un desafío clínico constante para el otorrinolaringólogo $0^{1,4}$.

Las causas de ES pueden dividirse en congénitas 0 adquiridas 5 . De éstas últimas, las secundarias a instrumentalización de la vía aérea siguen siendo las más frecuentes, con cerca del $90 \%$ de los ca$\operatorname{sos}^{1,2}$. Otras causas incluyen la granulomatosis de Wegener, sarcoidosis, infecciones granulomatosas de la vía aérea o por traumatismos laríngeos externos. No obstante, un importante número de ES son consideradas idiopáticas ${ }^{4}$.

Dentro de los factores de riesgo se han descrito una serie de características propias de la subglotis que la hacen más susceptible de presentar estenosis, como lo es su diámetro estrecho, su inextensibilidad, la fragilidad del tejido de revestimiento y una pobre vascularización ${ }^{6}$. Durante las últimas décadas un factor que ha sido fuertemente implicado es el reflujo laringo faríngeo (RFL). Este sería un elemento contribuyente, no así el causal, pero se recomienda su estudio y tratamiento si está presente, ya que su control simplifica y mejora el manejo de la estenosis ${ }^{1,4}$. Asimismo se describen infecciones, lesiones traumáticas de la vía aérea superior, isquemia, hipotensión, cirugías y el uso de láser como factores que pueden favorecer, perpetuar y agravar esta condición?.

Respecto de la sintomatología, generalmente no se presenta hasta alcanzar etapas avanzadas donde el principal síntoma es la disnea. Puede también manifestarse como estridor laríngeo, tos, sensación de falta de aire, disfonía, cianosis 0 molestias laríngeas ${ }^{2,4}$. Dado la dinámica del flujo aéreo, pequeñas variaciones en el diámetro de la vía respiratoria 0 en el largo de la estenosis son significativas para el flujo de aire, por tanto el paciente comenzará a manifestar episodios de disnea rápidamente progresiva ${ }^{8}$. Afortunadamente, los pacientes toleran bien cierto grado de estenosis, hasta $50 \%$ en ciertos casos. Pero el retraso en la consulta médica y la frecuente atribución de los síntomas a cuadros de asma o bronquitis, junto con múltiples tratamientos y evaluaciones recibidas por otras causas de disnea, hacen que la búsqueda de un agente causal sea casi imposible, además de retrasar la evaluación por parte del especialista ${ }^{2,4}$.

El diagnóstico de ES no siempre es sencillo. Dado lo inespecífico de los síntomas, es fundamental un alto índice de sospecha². Para la evaluación 
clínica son fundamentales las técnicas endoscópicas y los estudios de imágenes. La endoscopía flexible, y en especial la rígida, son las principales armas diagnósticas. Ellas permiten estimar la función laríngea, valorar la movilidad de las cuerdas vocales, ver el calibre de la vía aérea y localizar, ver la extensión y el estadio evolutivo de la estenosis $^{9,6}$. La tomografía computarizada helicoidal (CT) constituye el estudio más valioso en la evaluación prequirúrgica, dada su especificidad al determinar la extensión y el grado de severidad de la estenosis, especialmente mediante el uso de técnicas de reconstrucción 3D de la vía aérea y broncoscopía virtual ${ }^{4,6,8,10}$. La resonancia nuclear magnética (RNM) permite la captura de imágenes en todos los planos y define mejor la anatomía de la región ${ }^{11,7}$, no obstante, su interpretación no es tan clara como la CT y presenta un mayor costo asociado. Asimismo, una curva flujo volumen con un patrón obstructivo extratorácico y una radiografía lateral simple de cuello pueden ser orientadoras ${ }^{3,11}$.

Mediante esta evaluación se podrá definir el grado de estenosis. La clasificación más utilizada y de mayor aplicabilidad para determinar el tipo de manejo es la de Myer y Cotton de 1994, definida según el porcentaje de obstrucción del lumen de la vía aerea ${ }^{10,11}$.

Con respecto al tratamiento, éste sigue siendo un desafío. Debe ser individualizado según cada paciente y tipo de estenosis, donde el principal objetivo será restablecer un calibre suficiente en la vía aérea que permita respirar por la vía normal ${ }^{6}$.

Actualmente, existen 3 opciones terapéuticas aceptadas: la reconstrucción laringotraqueal y anastomosis (RLT), la laringoplastía (LP) sin resección segmentaria, con o sin injerto de hueso o cartílago, y procedimientos endoscópicos como la dilatación con balón, colocación de prótesis y láser entre otras ${ }^{6,11}$.

En consecuencia, por la complejidad de esta patología y al alto grado de morbimortalidad que conlleva, el presente estudio busca determinar el perfil de pacientes atendidos en nuestro servicio con diagnóstico de ES, describir las características demográficas (edad, sexo), antecedentes mórbidos, factores de riesgo asociados, sintomatología, tipo y grado de estenosis, tipo de tratamiento, tasa de éxito según el porcentaje de decanulación y complicaciones.

\section{OBJETIVO}

Determinar el perfil de pacientes con diagnóstico de estenosis subglótica, evaluar el tratamiento y sus resultados.

\section{MATERIAL Y MÉTODO}

Estudio de tipo retrospectivo descriptivo. Se incluyeron un total de 17 pacientes adultos con diagnóstico de ES atendidos en el Servicio de Otorrinolaringología del Hospital del Salvador (HDS) e Instituto Nacional del Tórax (INT) entre los años 2008 y 2011. A partir de la revisión de fichas clínicas, se registró la edad, sexo, antecedentes mórbidos, factores de riesgo asociados, sintomatología, tipo y grado de estenosis, tipo y número de tratamientos realizados, porcentaje de decanulación y complicaciones. Pacientes con diagnóstico de ES con fichas incompletas, ilegibles o con ausencia de alguna de las variables estudiadas fueron excluidos. Los datos obtenidos fueron rotulados en una planilla Excel y analizados con el programa SPSS versión 11.

\section{RESULTADOS}

Para el periodo estudiado, 17 pacientes adultos cumplieron con los criterios de inclusión. El promedio de edad general fue de $51 \pm 14,37$ años, con un rango de 17 a 79 años. En el caso de las mujeres, el promedio fue de $53,17 \pm 12,65$ años y $45,8 \pm 18,38$ años para Ios hombres. El 70,6\% de los pacientes correspondió al sexo femenino y el $29,4 \%$ al masculino.

De los antecedentes mórbidos, el $12 \%$ de los pacientes presentaba RGE, 17\% TEC, 18\% mesenquimopatías, el $25 \%$ no los presentaba al momento

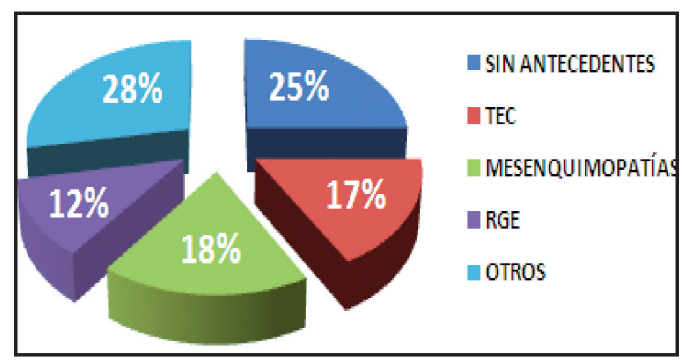

Figura 1. Antecedentes mórbidos. 
del diagnóstico y $28 \%$ presentaba otros antecedentes no relacionados con la ES (Figura 1).

Con respecto a los factores de riesgo asociados a ES, el $100 \%$ de los pacientes no los presentaba. En cuanto a las causas de estenosis subglótica previo al estudio de los pacientes, 5 de ellos tenía el antecedente de intubación prolongada, 1 de estenosis congénita y 1 el diagnóstico de granulomatosis de Wegener (Tabla 1).

En relación a la sintomatología clínica, la disnea fue el principal síntoma afectando al $76,5 \%$ de los pacientes, seguido de estridor laríngeo, siendo la tos y la disfonía manifestaciones menos frecuentes (Tabla 2). La mediana de tiempo entre el inicio de los síntomas y la fecha del diagnóstico fue de 10 meses. Para el diagnóstico, al $100 \%$ de los pacientes se les realizó una evaluación endoscópica y una tomografía computarizada de cuello. Estudios funcionales fueron realizados en el $47,1 \%(8 / 17)$ de los casos. En todos ellos se encontró como hallazgo una obstrucción fija extratorácica. Respecto al tipo de estenosis encontrada, el 88,2\% de los pacientes presentó una estenosis subglótica de partes blandas, sin compromiso del cartílago (Tabla 3). Según el grado de severidad de Myer y Cotton, el III grado es el predominante en toda la serie, seguido del grado II. No obstante, en un porcentaje no menor de los casos, no se describieron los hallazgos mediante el uso de esta escala de clasificación (Figura 2).

Referente al manejo y tipo de tratamiento, se emplearon tanto procedimientos endoscópicos (dilatación con broncoscopio) como quirúrgicos abiertos (resección laringo traqueal) en similares porcentajes, siendo necesario utilizar ambas terapias solamente en 2 pacientes (Figura 3).

La mediana de procedimientos fue de 2 con un rango de 1 a 4 , siendo el $100 \%$ de éstos bajo anestesia general. La mediana de seguimiento de la serie fue de 14 meses con un rango de 12 a 36 meses.

En cuanto a la tasa de éxito, expresada como porcentaje de decanulación, ésta fue de 58,8\%. Si bien un porcentaje no menor de pacientes aún persiste con traqueotomía $(41,2 \%)$, al separar los resultados por tipo de procedimiento, vemos que aquellos pacientes sometidos a tratamiento endoscópico o cirugía abierta como segundo paso, presentan un mayor porcentaje de decanulación en relación a aquellos sometidos sólo a cirugía abierta (Figura 4). Finalmente, en relación a las complicaciones, éstas afectaron al $41,7 \%$ de los pacientes.
Tabla 1. Causas de estenosis subglótica previo al estudio

\begin{tabular}{|l|c|c|}
\hline Causa & $\begin{array}{c}\text { Frecuencia } \\
\text { absoluta }\end{array}$ & $\begin{array}{c}\text { Frecuencia } \\
\text { relativa }\end{array}$ \\
\hline $\begin{array}{l}\text { Intubación } \\
\text { prolongada }\end{array}$ & $5 / 17$ & $29,4 \%$ \\
\hline $\begin{array}{l}\text { Granulomatosis de } \\
\text { Wegener }\end{array}$ & $1 / 17$ & $5,9 \%$ \\
\hline Estenosis congénita & $1 / 17$ & $5,9 \%$ \\
\hline
\end{tabular}

Tabla 2. Sintomatología

\begin{tabular}{|l|c|c|}
\hline Síntomas & $\begin{array}{c}\text { Frecuencia } \\
\text { absoluta }\end{array}$ & $\begin{array}{c}\text { Frecuencia } \\
\text { relativa }\end{array}$ \\
\hline & & \\
\hline Disnea & $13 / 17$ & $76,5 \%$ \\
\hline Estridor & $9 / 17$ & $52,9 \%$ \\
\hline Tos & $5 / 17$ & $29,4 \%$ \\
\hline Disfonía & $1 / 17$ & $5,9 \%$ \\
\hline N/E & $1 / 17$ & $5,9 \%$ \\
\hline
\end{tabular}

Tabla 3. Tipo de estenosis

\begin{tabular}{|l|c|c|}
\hline $\begin{array}{l}\text { Tipo de } \\
\text { estenosis }\end{array}$ & $\begin{array}{c}\text { Frecuencia } \\
\text { absoluta }\end{array}$ & $\begin{array}{c}\text { Frecuencia } \\
\text { relativa }\end{array}$ \\
\hline $\begin{array}{l}\text { Partes } \\
\text { blandas }\end{array}$ & 15 & $88,2 \%$ \\
\hline $\begin{array}{l}\text { Cartilaginosa } \\
\text { (mixta) }\end{array}$ & 2 & $11,8 \%$ \\
\hline
\end{tabular}

Tabla 4. Complicaciones

\begin{tabular}{|l|l|}
\hline 1 & Fístula tráqueo-cutánea \\
\hline 3 & Reestenosis \\
\hline 1 & Enfisema subcutáneo \\
\hline 1 & Sinequia de cuerda vocal (CV) \\
\hline 1 & Neumonía \\
\hline 1 & Bronquitis posquirúrgica \\
\hline 1 & $\begin{array}{l}\text { Paciente con numerosas complicaciones } \\
\text { además de la reestenosis: parálisis de } \\
\text { CV, granulomas y dehiscencia de herida } \\
\text { operatoria }\end{array}$ \\
\hline
\end{tabular}

El detalle se describe en la Tabla 4. 


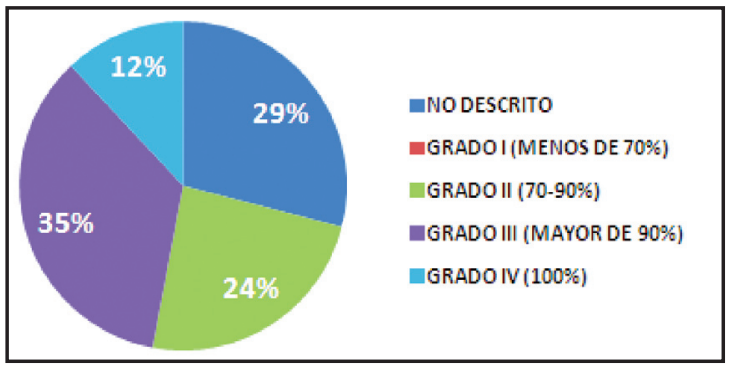

Figura 2. Grados de severidad de estenosis de Cotton.

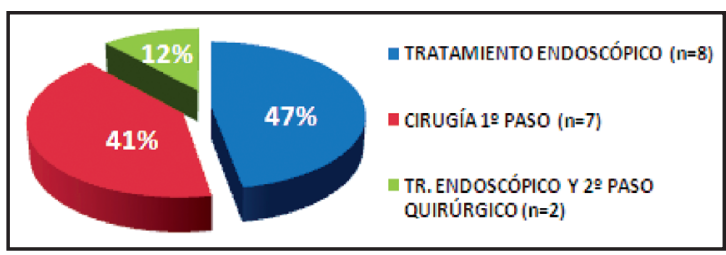

Figura 3. Tipo de tratamiento.

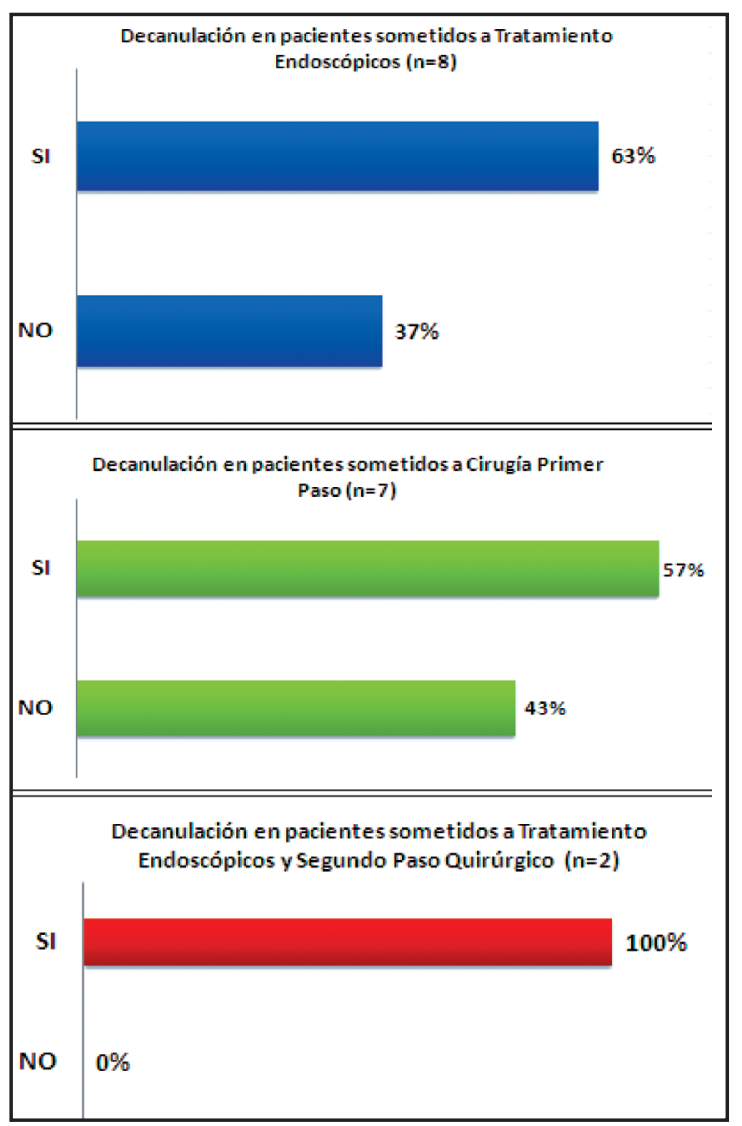

Figura 4. Porcentaje de decanulación según tipo de tratamiento.

\section{DISCUSIÓN}

En el adulto la ES es una patología poco frecuente, cuya etiología se relaciona con causas traumáticas, infecciosas, neurológicas, neoplásicas 0 inmunológicas ${ }^{6}$.

En la presente serie de pacientes, al momento del diagnóstico sólo el 41,2\% (7/17) de ellos contaba con una causa identificada de estenosis determinada por el antecedente de intubación prolongada, de granulomatosis de Wegener y estenosis congénita. Posterior al estudio y manejo, fue posible la identificación de 2 casos más de Wegener a través de exámenes reumatológicos, aumentando al 52,9\% la certeza diagnóstica, siendo el resto de los casos definidos como idiopáticos.

En éstos últimos, al igual que otras series, se mostró una predominancia de pacientes del sexo femenino ${ }^{4}$.

En relación a las distintas opciones de tratamiento, en nuestra experiencia, la técnica quirúrgica abierta realizada como único tratamiento o como segundo paso tras el fracaso de un procedimiento endoscópico, presentó un éxito satisfactorio en el resultado quirúrgico, con escasa morbilidad y nula mortalidad similar a lo descrito en la literatura ${ }^{11}$. Sin embargo, resultó ser menos exitosa en comparación a la terapia endoscópica, presentando un porcentaje de decanulación menor al descrito para esta técnica el que puede alcanzar un $90 \%$ 6,11. Esto puede deberse al mayor grado y extensión de la estenosis de los pacientes seleccionados para esta técnica, versus aquellos sometidos a tratamiento endoscópico, en quienes la severidad de la estenosis fue menor.

Como procedimiento endoscópico la técnica seleccionada fue la dilatación con broncoscopio. La tasa de éxito para el tratamiento endoluminal, según diversos reportes, varía entre 42\%-82\% según la extensión de la estenosis, siendo mejor en estenosis menores de $1 \mathrm{~cm}$ y sin destrucción estructural ${ }^{11}$. En los pacientes en quienes se utilizó esta técnica (47\%), la tasa de éxito obtenida de $63 \%$ se encuentra dentro de los rangos descritos.

Respecto a la laringoplastía, es una técnica que requiere de varias etapas y la tasa de 
éxito reportada con y sin injerto es de $76 \%{ }^{11}$. Sin embargo, para esta serie de casos no existe mayor experiencia durante los años analizados.

Como limitaciones podemos mencionar que el tipo de diseño utilizado presenta desventajas dado su carácter retrospectivo y un mayor riesgo de sesgo. Asimismo, la exclusión de pacientes pediátricos pudiese alterar los resultados obtenidos al modificarse los porcentajes de las variables estudiadas.

\section{CONCLUSIÓN}

La ES es una patología compleja. Su baja tasa de sospecha, lo inespecífico de sus síntomas y el retraso en la consulta médica hacen necesaria la creación de equipos de vía aérea multidisciplinarios y en formación continua para lograr la pesquisa en estadios precoces de su evolución. El manejo deber ser caso a caso y el control del RFL/RGE, de estar presente, debe ser parte del tratamiento habitual para simplificar y mejorar la resolución de la estenosis. Del mismo modo, es necesaria la creación de protocolos de diagnóstico y tratamiento estandarizados que incluyan estudios de imágenes, procedimientos endoscópicos, evaluaciones funcionales y la búsqueda activa de la causa subyacente que explique la estenosis y oriente al manejo posterior.

\section{BIBLIOGRAFÍA}

1. Lorenz R. Adult Laryngotracheal Stenosis: Etiology and Surgical Management. Adult Laryngotracheal Stenosis: Etiology and Surgical Management. Curr Opin Otolaryngol Head Neck Surg 2003; 11(6): 467-72.
2. Scholz A, Srinivas K, Stacey M, Clyburn P. Subglottic Stenosis in Pregnancy. British Journal of Anaesthesia 2008; 100 (3): 385-8.

3. Blaivas AJ, Strauss W, Yudd M. Subglottic Stenosis as a Complication of Wegener's Granulomatosis. Prim Care Respir J 2008; 17(2): 114-6.

4. Blumin J, Johnston N. Evidence of Extraesophageal Reflux in Idiopathic Subglottic Stenosis. The Laryngoscope 2011; 121: 1266-73.

5. Krimsky W, Rodrigues M, Malayaman N, Sarkar S. Spray Cryotherapy for the Treatment of Glottic and Subglottic Stenosis. Case Report. The Laryngoscope 2010; 120: 473-7.

6. Alvarez-Buylla M, Vázquez J, González J, Mártinez J. Tratamiento quirúrgico de las estenosis subglóticas. Acta Otorrinolaringol Esp 2010; 61: 282-6-vol.61 núm 04.

7. Singh T, Sandulache V, Otteson T, Barsic M, Klein E, Dohar J, HeBda P. Subglottic stenosis examined as a fibrotic response to airway injury characterized by altered mucosal fibroblast activity. Arch Otolaryngol Head Neck Surg 2010; vol 136 No. 2.

8. SchweInfuRTH J. Endoscopic Treatment of Severe Tracheal Stenosis. Ann Otol Rhinol Laryngol 2006; 115(1): 30-4.

9. Ortega C, Ortega P, Cervera J, Asgharpour E. (2005). Estenosis Laríngeas en Algarra J. Tratamiento de las Vías Respiratorias. (117-123), Barcelona: Masson.

10. Contreras JM, Paredes A, Niklas L, Changhua Lu, Contreras P. Estenosis Laringotraqueal. Experiencia Clínica. Rev Otorrinolaringol Cir Cabeza Cuello 2011; 71: 107-16.

11. Yamamoto K, Kojima F, Tomiyama K, Nakamura T, HaYASHIno Y. Meta-Analysis of Therapeutic Procedures for Acquired Subglottic Stenosis in Adults. Ann Thorac Surg 2011; 91: 1747-53. 